\title{
3D scene capture: a comprehensive review of techniques and tools for efficient Life Cycle Analysis (LCA) and Emergency Preparedness (EP) applications
}

\author{
F. Fadli ${ }^{1}$, H. Barki ${ }^{1}$, P. Boguslawski ${ }^{2}$ \& L. Mahdjoubi ${ }^{2}$ \\ ${ }^{1}$ Department of Architecture and Urban Planning, \\ College of Engineering, Qatar University, Qatar \\ ${ }^{2}$ Department of Architecture and the Built Environment, \\ University of the West of England, UK
}

\begin{abstract}
3D scene capture is a critical task in many domains, especially for BIM model reconstruction of existing facilities, Life Cycle Analysis (LCA), and Emergency Preparedness (EP). While initial CAD/GIS plans and drawings, if they exist, represent a valuable source of information, acquiring knowledge about the dynamic scene, aspects like crowd movement or furniture is a necessary and difficult task. In this context, 3D capture becomes unavoidable as it is the only way to deal with such dynamic information. We propose, in this work, a critical review of 3D capture techniques and tools, ranging from photogrammetry to 3D scanning. Our study distinguishes itself by being oriented towards 3D capture practitioners, who need to make critical decisions about the choice of adequate acquisition technologies for a particular application. We review 3D capture techniques by exposing their pros and cons, and discuss the most relevant aspects of each technology, like equipment/operation costs, mobility, ease of use, learning curve, acquisition accuracy, precision and range, generated data complexities, post-processing considerations, and applications. The paper aims at synthesizing the analysis by developing a set of recommendations for the use of 3D capture in various contexts with special reference to LCA and EP in the built environment.
\end{abstract} Keywords: $3 D$ capture, laser scanning, photogrammetry, BIM model reconstruction, $L C A$. 


\section{Introduction}

$3 \mathrm{D}$ capture is the process of generating 3D models from sensor data dispatched over a scene. With the recent progress made in computing hardware, especially in computer graphics and data acquisition technologies, and the rapidly decreasing prices of such equipment, 3D capture has become more accessible for professionals and even for amateurs. It is now possible to create and manipulate large amounts of very complex 3D data, such as cloud points and meshes, describing the geometry, topology, texture, and other physical properties of a surrounding scene, all this can be done in cost and time-efficient ways.

3D capture is considered as a critical task for many application domains, like BIM, building surveying, robot motion planning, life cycle analysis [1], and emergency preparedness [2]. It is essential as it constitutes the first step towards the development of suitable indoor/outdoor BIM models that greatly help practitioners by offering better visualization and interaction means. While initial CAD/GIS plans, if they exist for a particular scene, represent a valuable source of information; acquiring knowledge about dynamic scene aspects (human behaviour, construction and as-built differences) is a necessary and relatively difficult task, making 3D capture unavoidable in our context, because it is the only way to deal with dynamic scenes information.

In this paper, we propose a critical review of the available $3 \mathrm{D}$ capture techniques and their potential, ranging from photogrammetry to $3 \mathrm{D}$ scanning. While 3D capture techniques have already been studied in the literature, most of the existing discussions tend to summarize the literature, do not discuss some relevant aspects, or do not provide a thorough analysis helping practitioners in making relevant decisions about the adequate technology for the targeted application domain. Our comprehensive analysis distinguishes itself from previous works in the sense that it is oriented towards $3 \mathrm{D}$ capture practitioners who need to make such critical decisions, by examining the relevant aspects of each technology, the different pros and cons, and the potential application domains for each technique. 3D capture techniques are analysed and compared according to many aspects that are important to field practitioners, like equipment and operation costs, mobility, flexibility and ease of use, learning curve, acquisition accuracy, precision and range, temporal and spatial complexities of generated data, post-processing considerations, applications, etc. We also provide a set of recommendations for field practitioners in order to help adopting the most adequate $3 \mathrm{D}$ capture technologies in various contexts.

The rest of the paper is organized as follows: in section 2 we briefly expose the different techniques proposed in the literature for the 3D acquisition of scenes, with a focus on the most prominent ones: photogrammetry and laser scanning. In section 3, we provide a comprehensive comparison of these techniques by considering the aforementioned aspects. Finally in section 4 we develop a set of recommendations for field practitioners in order to help them in choosing the more adequate $3 \mathrm{D}$ capture technique(s), according to the application context. 


\section{Overview of 3D scene capture techniques}

The state of the art of 3D capture shows that several techniques have been developed and used for 3D data acquisition. A strict classification of these techniques may not be possible but one may talk about two broad categories: 3D scanning/modelling and image-based techniques.

The first and most basic technique relied on manually measuring the geometry of a scene and translating it through drafting boards into paper-based drawings. Later on, the CAD modelling (cf. Figure 1) has emerged as a consequence of the development of CAD tools and allowed the generation of 3D models. However, such methods require long modeling times for complex scenes, are unsuitable for the reproduction of fine or highly curved architectural details, necessitate highly skilled operators, and induce a loss of control on the accuracy of the generated model.
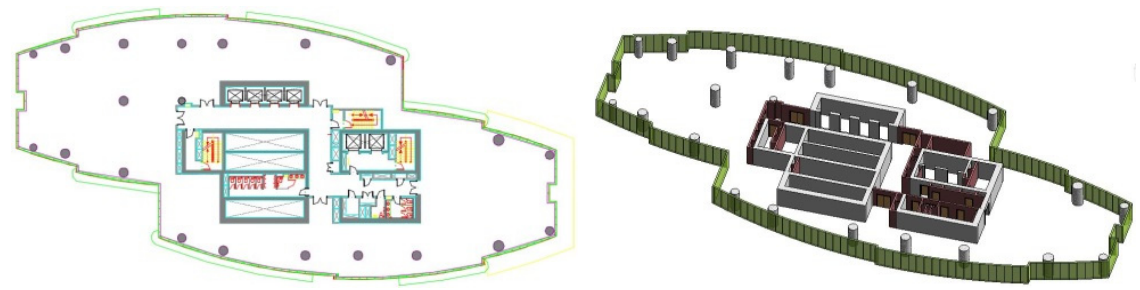

Figure 1: CAD modeling illustration for a floor of a skyscraper building. Left: 2D CAD model. Right: 3D BIM model.

Thanks to the data acquisition hardware recent developments, 3D scanning becomes more affordable. According to different criteria [3], one may classify 3D scanning techniques into different categories, such as contact/non-contact, passive/active, reflective/transmissive, destructive/non-destructive, optical/nonoptical, etc. Active and contact-based probing techniques have been employed to capture the shape of 3D physical objects and generate accurate 3D models. These techniques use Coordinate Measuring Machines (CMM) composed of mechanical arms that touch the surface of objects along user-defined profiles (cf. Figure 2 left), so that contact-points' coordinates can be deduced from the moving configurations of the probe arms [4]. Although successfully used for reverse engineering, such a time-consuming and manually operated technique does not provide consistent control on the sampling accuracy, does not allow recording visual properties of objects, and doesn't work on soft or large-size objects (destructive approach).

Non-contact 3D scanning techniques, either optical, e.g. Lidar, or non-optical, e.g. Radar, Sonar, or Computer Tomography (CT) employ different remote sensing principles (Laser light for Lidar, microwaves for Radar, etc.). Such techniques may be classified into transmissive and reflective ones, depending on the nature of the interaction of the emitted wave with the target objects. These techniques do not intrinsically interfere with the scanned object, reducing the destructive impact of the capture and allowing to operate on fragile objects. They 

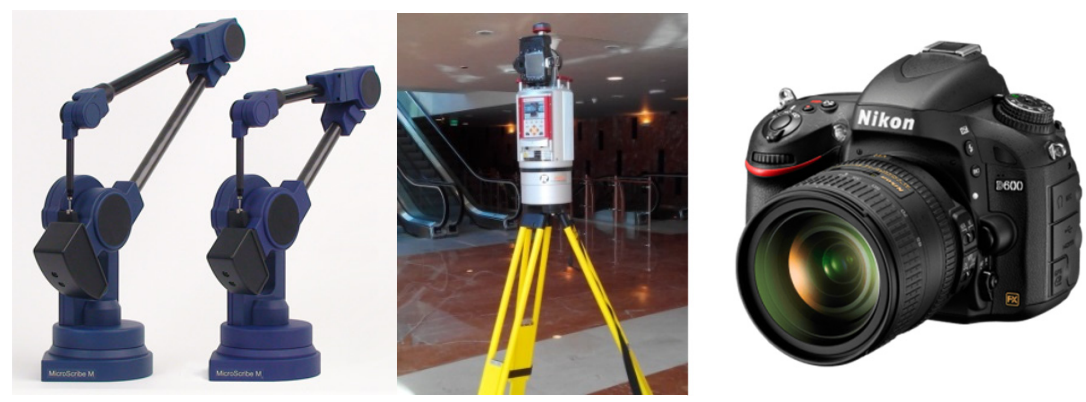

Figure 2: 3D scanning/photogrammetry devices. Left: A contact-based MicroScribe MX device (photo taken from [4]). Center: The Riegl VZ-400 terrestrial laser scanner. Right: The Nikon D600 digital camera shipped with Riegl VZ-400 scanner.

also widen the capture range as no physical contact is required. Lidar or laser scanning technique is the most relevant in our context. The principle consists in emitting laser beams, of frequencies typically between 500-1500 nm [5], and analysing the reflected beams, in order to measure the distance between the device (cf. Figure 2 center) and the scanned objects. Laser scanners are widely used as we will see next. One of the main reasons of their wide adoption is that a laser beam has a tight focus, meaning that it can be used to capture large scenes, contrary to other optical techniques which lose focus for large distances.

Image-based techniques and especially photogrammetry (also known as stereoscopy) have a long history as they find their roots in the middle of the 19th century. According to [6], Aimé Laussedat (a French inventor) is the first one who thought of using still images for mapping purposes. However, it is only recently that the availability of high quality cameras (cf. Figure 2 right) encouraged the use of photogrammetry to model 3D scenes. Photogrammetry aims to produce measures of a scene and to deduce its 3D structure by examining a set of images. The principle consists in positioning targets whose coordinates are known in advance on the objects to be captured, and then taking several image captures of these objects from different positions and angles. By using the a priori information of the camera positions/rotations, the target points coordinates, and some principles of projective geometry, the captured images are combined to form a $3 \mathrm{D}$ model of the scene [7]. One of the advantages of photogrammetry is that it allows extracting colour and texture information. However, in extreme conditions (low textures or very complex objects), photogrammetry reaches its limits. Photogrammetry may be used effectively as a complement to 3D laser scanning and the following sections provide usage recommendations.

\section{Review of 3D capture technologies}

The aim of the current discussion is to compare the most prominent capture techniques from the aforementioned two categories (image-based and 3D 
scanning). This non-exhaustive discussion focuses on photogrammetry and laser scanning, but whenever relevant, some of their variations are mentioned.

\subsection{Safety and autonomy/mobility}

Photogrammetric techniques only require the use of conventional still cameras after the placement of identified targets. Therefore, the process is not harmful for the operator, contrary to laser scanning which is harmful for the operator's eyes. Recently, there is a trend of using LED light in 3D scanning as a replacement of laser. The advantage is that LED light is safer and as accurate as laser light (for close range captures only). When it comes to autonomy of capture devices, photogrammetry requires less power consumption than laser, as no built-in amplifier is required as in laser scanners, enabling the production of more autonomous and mobile capture devices. We shall however mention that some hardware manufacturers recently propose handheld flexible laser scanners which are primarily used for scanning small size objects and attempts have been made to use them for large scene capture.

\subsection{Equipment and operation costs}

Evaluating the capture budget is a crucial factor as this budget must fit project's financial constraints. Regarding equipment cost, photogrammetric devices are the most accessible ones, as they are basically manufactured for general public. While the prices are rapidly decreasing, the performance and specifications are continuously increasing, allowing them to be widely and easily used by ordinary people. Photogrammetric devices can be purchased for prices starting form tens of dollars only! On the other hand, despite the fact that laser scanners have been commercialized since three decades, the prices are still high and their decreasing evolution didn't follow the rapid drop of the other electronic devices prices. According to [3], laser scanners prices range from tens of thousands of dollars to hundreds of thousands of dollars, depending on the sophistication of the scanner, the included accessories/software, and the specifications. In consequence, laser scanners are still inaccessible for general public and are more tailored to companies or educational institutions with consequent budgets. A list of laser scanner providers may be found in [3].

Recently, some companies started proposing scanner rental services [8], as a compromise to the high purchase pricing. Besides budget considerations, it is also crucial to take into account the lifetime of the scanning device. While photogrammetric devices may be used for decades, laser scanners have a much smaller lifetime expressed in thousands of hours [5], because they are quickly deteriorated by the operational temperature of the built-in amplifier of the laser equipment.

Structural light scanners [9] represent another kind of 3D scanners that deserve a discussion. Unlike laser scanners, the principle is to project patterns of light (usually lines) and to use camera(s) for the capture of the light distortions. Specific software is then used to reconstruct the geometry of the exposed object from the light pattern distortions, yielding a 3D model. Structured light scanners are 
cheaper and may constitute an alternative to laser scanner in some contexts (tight budgets). However, they present weaknesses like loss of focus for large scenes, low resolution/accuracy, the need for separate emission/reception devices, and the manual handling of the capture process, contrary to laser scanners which require less user input. A popular example of a handheld structured light (infrared) scanner is the Kinect device shipped with the Microsoft Xbox gaming system.

Regarding the operational cost of a 3D capture process (equivalently the learning curve), since photogrammetric devices can be found on almost any private office, it is expected that ordinary people can use them easily. Laser scanners are not public-friendly and need specific trainings, so an additional operational cost has to be accounted (steep learning curve).

\subsection{Software}

Besides the aforementioned equipment cost considerations, the availability of cheap or open source software is one of the main points that influence the decision to adopt a capture technology. There exists a big gap between photogrammetry and laser scanning in terms of software support. Because laser scanner providers are mainly targeting professionals with consequent budgets, most of them provide specific software which is generally highly expensive and compatible only with the scanner it ships with. In extreme cases, it cannot be even used with other devices from the same company! Such software has closed source code, disabling the possibility of examining it for further R\&D purposes.

We shall note that even if each company has a proprietary closed file format, some open file formats allowing basic data exchange between different devices do exist. An example of proprietary laser scanning software is the RiSCAN PRO delivered with RIEGL devices (cf. Figure 2 center), which allows both remotely operating on the scanner and processing the captured data (filtering, registration, hole closing, resampling, etc.).

Because photogrammetric techniques emerged a long time before laser scanning, in addition to the availability of cheap price still cameras and their wide usage among general public, photogrammetric software offer is richer, cheaper, and even multiplatform. Autodesk 123D Catch is a freemium mobile application for hobbyists, which allows generating 3D models on the cloud from a set of overlapping images. Eos systems Photomodeler is well-known and probably the most mature and affordable commercial software (price starting from \$1145) using close-range photogrammetry for 3D models reconstruction. Other free and open source alternatives do exist like the openMVG C++ library and VisualSFM, making them good candidates for research purposes. Compared to laser scanning software, photogrammetric software is cheaper, more accessible, and relatively usable with any capture device.

\subsection{Resolution, precision, range, and capture details}

The quality a measurement system in general and of a 3D capture device in particular is usually assessed through a set of objective measures, among which we will define and discuss the range, resolution, precision, and accuracy 
parameters. The range of a capture device measures the maximal distance up to which a device can properly acquire the properties of an object located at that distance from the device. Resolution is a measure intrinsic to the capture device, as it measures the minimal distance between two distinct data samples (point in the case of laser scanning and pixels in the case of photogrammetry) and is sometimes called or inter-sample distance. Accuracy refers to the difference between the measured sample coordinates and the real physical coordinates of that sample in the captured scene. These parameters are not independent from each other, as for example, the resolution/accuracy of a data sample depends on the range or the distance of the corresponding physical sample from the capture device.

The accuracy of a laser scanning capture may be easily estimated in advance, compared to photogrammetry whose outcome is unpredictable because of many parameters, like the $2 \mathrm{D}$ image to $3 \mathrm{D}$ model conversion errors. Although some previous work claims that photogrammetry, especially with the increasing capabilities of imaging devices, can achieve similar or even higher resolution/accuracy than laser scanners, there is an agreement in the literature that laser scanning performs better in general and can go below the millimetre accuracy, and that for complex geometry scenes and objects, photogrammetric methods are still unable to reproduce accurate $3 \mathrm{D}$ models, compared to laser scanners [3]. Another factor consolidating these facts resides in the fact that laser beams have tight focus, meaning that they are more precise in capturing scenes at higher ranges, and even at very short ranges at the level of molecules [10]. For concrete measures of such parameters, the reader is referred to the manuals chipped with the different capture devices.

Another aspect distinguishing photogrammetry and laser scanning techniques capture details resides in the environmental conditions under which these techniques are guaranteed to perform correctly. Because of its emissive nature, laser scanning is less affected by ambient light changes (day or night operations) and the resulting acquisition data is relatively invariant with respect to climate conditions. However, because of their properties, very shiny materials like water surfaces are impossible to scan. On the other hand, photogrammetric capture is highly influenced by weather/light fluctuations, and the capture outcome highly depends on the scene illumination, as the capture result deteriorates for dark scene areas. This point represents one of the main weaknesses of photogrammetry.

\subsection{Temporal/spatial data complexity and processing}

Laser scanning is the more advanced data capture. Compared to photogrammetry, laser scanning has shown to be more efficient in capturing data quickly, as millions of points can be captured per second and this rate is even increasing. The main reasons for this high capture speed reside in the fact that scanners provide an automated way of scanning large 3D areas (at least for a single scan) in 360 horizontal direction, by using tight focus laser beams, allowing for more capture density. On the other hand, photogrammetry relies on 2D capture and then on some heavy processing for $3 \mathrm{D}$ point cloud generation, making it less efficient and constrained by the single image capture resolution. Laser scanning can capture 
data in near real-time, compared to photogrammetry, which is an offline process. We shall note that in order for efficiency claims to be fair, the same data set have to be targeted for both photogrammetry and laser scanning. In the literature, laser scanning has been reported to be slow compared to photogrammetry when using high resolutions, but this is not true as if one wants to target the same resolution with photogrammetry, this is sometimes impossible and if it is possible, it makes photogrammetric capture slower.

Because of the speed of 3D laser capture, it is expected that the spatial complexity of the acquired data is more consequent than that of photogrammetry. Depending on the resolution of the scanner, the more laser beams are emitted or projected onto a scene, the more points are collected and typical point clouds may easily attain billions of points for large projects where hundreds of scans may be required. Such generated point clouds may provide rich and very detailed information about a scene, but a lot of information makes the task of processing, analysing, and extracting pertinent knowledge more difficult. Detailed point clouds correspond to large size (tens to hundreds of gigabytes) and complex files which require high computation power and are extremely difficult to handle by processing software. Photogrammetric data is less cumbersome than laser data. However, with the progress made in of imaging device specifications and algorithms, current applications may involve tens of thousands of images yielding to very large point clouds.

For photogrammetry, the most critical issue concerns processing or combining individual images into a 3D model. The placement of targets allowing individual image registration and the manual choice of individual image spatial positions/rotations represent time consuming and tedious tasks especially if the number of snapshots/target points increases. The most time consuming sub-step in a photogrammetric process is the combination of the individual $2 \mathrm{D}$ images into a unique $3 \mathrm{D}$ point cloud. On the other hand, such a resource consuming $2 \mathrm{D}$-to-3D conversion sub-step is unnecessary in laser scanning, as data is already in $3 \mathrm{D}$ form and the registration of the individual $3 \mathrm{D}$ point clouds is relatively easier.

The reader interested in precise measures of $3 \mathrm{D}$ capture complexity and processing cost may refer to specific papers [11] and capture hardware manuals [12]. We avoided mentioning precise numbers because they tend to change from a hardware/software system to another and are also customizable within the same device.

\subsection{Applications}

Whenever some geometric or physical information about a scene is required or needs to be reconstructed, data capture enters into action. Photogrammetry and 3D scanning have been interchangeably and successfully used in many applications. On the one hand, Terrestrial Laser Scanning (TLS) has been applied for interior building modeling, navigation, and exploration [13], while Airborne Laser Scanning (ALS) has been used for 3D city/terrain modelling and landslide volume computation in geology, in order to capture the geometry of cities and terrains [14]. In transportation projects, it has been used for acquiring design and construction data [11]. Cultural heritage and historical buildings digitization is 
probably the most explored domain where laser capture has been used for heritage documentation and preservation [13]. On the other hand, photogrammetry touched similar application domains like for example in bridge engineering [15], but the application domain that deserved most of the researchers attention was cultural heritage preservation, where it has been used for the digitization and reconstruction of photorealistic 3D models for many historical sites [7, 16], thanks to the ability of photogrammetry to better capture visual aspects of scenes.

\section{Recommendations for field practitioners}

The provided review reveals that laser scanning technology represents the future of $3 \mathrm{D}$ capture. It is the most promising $3 \mathrm{D}$ capture tool as it is the most accurate one. On the other hand, photogrammetry provides less complex data than laser scanning, but requires further costly processing of that data. The accuracy of the resulting 3D models is limited by the images' accuracy, as well as the precision of the registration of the different images.

As predicted by Debevec [6] in the beginning of the 21 st century, since each technology comes with its own set of limitations, it is interesting to look towards combining different technologies, especially laser scanning and photogrammetry, in order to improve the accuracy of photogrammetry and to reduce or ideally eliminate the manual steps required for generating 3D models. Nowadays, the trend is going towards such a combined use of techniques for better reconstruction and modeling outcomes. As an example of such an integration of capture techniques, in the LCA context, El-Omari and Moselhi [1] described a progress reporting application, where both photogrammetry and laser scanning have been combined to improve the accuracy and speed of collecting data from a construction site. In cultural heritage digitization, laser scanning and photogrammetry have been recently used in conjunction for developing documentation systems for "Umayyad" desert palaces in Jordan [16], "Quattro Canti" historical monument of Palermo [17], and Fatih mosque of Istanbul [18], etc. In robot motion planning, laser and vision sensors were combined for the development of a robot navigation system in indoor environment [19].

We shall note that besides the aforementioned conventional techniques, new data capture tools have emerged recently, thanks to the miniaturization of electronic devices, the development of new communication standards, and the dramatic drop of electronic chip prices. Such techniques include Radio Frequency Identification (RFID), Bluetooth, and bar coding. They may be used for some specific data capture subtasks in a more cost/time-effective way than laser scanning or photogrammetry. For instance, RFID may be used to track crowd movement within a facility in the context of an EP scenario, by providing pertinent and concise data about tracked people (their position and even identification in the building), while laser scanning and photogrammetry, will generate a lot of unnecessary data which is time/space complex, needs costly processing to extract pertinent knowledge equivalent to what RFID offers, and inhibits real-time operating which is a critical requirement for EP applications. 
As a synthesis for our discussion, the previous discussions emphasize that there is not ideal 3D capture technique to be used in all circumstances, as each technique offers advantages but comes with inherent problems. No one of the exposed techniques can be universally considered better than the others. When the need arises for data capture in a particular context, one has to first carefully identify the application needs in terms of data capture and to transpose them to each technique by considering its strengths and weakness, in order to find the (relatively) most adequate one. As most often, no technique perfectly fits a particular application context; one might consider using other techniques, in conjunction or complementation of the first one, in order to improve the capture and extraction of relevant knowledge. In cultural heritage, it appears that combining laser scanning (more precision) and photogrammetry (better visualization) gives the best results, while in an EP context, laser scanning and RFID represent good candidates as laser scanning, when used offline, enables precisely acquiring the 3D geometry of the facility, while RFID enables tracking people in a real-time way.

In future, we believe that the main challenges that researchers need to address for 3D capture consist in:

- $\quad$ Reducing the time required to perform a capture campaign, especially when high resolutions are used.

- Determining the minimum number of points/images sufficient for extracting the needed scene information.

- Developing efficient tools for the extraction of semantics from the raw captured data.

\section{Conclusions}

The paper develops a comprehensive review of 3D capture techniques and tools, with a deliberate focus on the most prominent ones: 3D laser scanning and photogrammetry. These techniques have been introduced and compared by exposing their weaknesses and strengths, according to many criteria of relevance for 3D capture practitioners like equipment/operation costs, mobility, accuracy, precision and range, data complexities, etc.

As 3D scene capture touches a plenty of application domains, this work targets a wide audience of professionals. It provides a set of recommendations and advices that help data capture actors for the correct and critical choice of adequate 3D capture technologies that best suit the targeted application, with special reference to LCA and EP in the built environment.

Even if our study gives some hints facilitating the choice of adequate 3D capture tools, it shows that a perfect or an ideal technology may not exist for a particular application domain, but any technology that reduces the tedious manual subtasks involved in 3D capture is more than welcome and non-conventional techniques like RFID should also be investigated. While laser scanning excels in accurately capturing the geometry of scene objects, photogrammetry excels in extracting visual details like texture and colours, making it a complementary 
technology and motivating the integration of different capture techniques for better results.

\section{Acknowledgement}

This research/publication was made possible by a National Priority Research Program NPRP award (NPRP-06-1208-2-492) from the Qatar National Research Fund (a member of The Qatar Foundation). The statements made herein are solely the responsibility of the author(s).

\section{References}

[1] El-Omari, S. \& Moselhi, O., Integrating 3D laser scanning and photogrammetry for progress measurement of construction work. Automation in Construction, 18(1), pp. 1-9, 2008.

[2] Barki, H., Fadli, F., Boguslawski, P., Mahdjoubi, L. \& Shaat, A., BIM models generation from 2D CAD drawings and 3D scans: an analysis of challenges and opportunities for AEC practitioners. Proceedings of the International Conference on Building Information Modelling (BIM) in Design, Constructions and Operations, 2015.

[3] Scopigno, R., Tutorial T1: 3D Data Acquisition. EUROGRAPHCS'02 Tutorials, 2002.

[4] MicroScribe Portable Scanners, http://www.3d-microscribe.com/

[5] Baltsavias, E.P., A comparison between photogrammetry and laser scanning. ISPRS Journal of Photogrammetry and Remote Sensing, 54(2-3), pp. 83-94, 1999.

[6] Masson, B.T., Paul Debevec and the Art of Photogrammetry. VFX-Procom, pp. 2-4, 2000.

[7] Debevec, P.E., Taylor, C.J. \& Malik, J., Modeling and rendering architecture from photographs. Proceedings of the 23rd annual conference on Computer graphics and interactive techniques - SIGGRAPH '96, ACM Press: New York, USA, pp. 11-20, 1996.

[8] Laser scanning Europe. Rent a laser scanner at little cost, http://www.laserscanning-europe.com/en/rent-laser-scanner/rent-laserscanner-little-cost.

[9] Ritz, M., Langguth, F., Scholz, M., Goesele, M. \& Stork, A., High resolution acquisition of detailed surfaces with lens-shifted structured light. Computers \& Graphics, 36(1), pp. 16-27, 2012.

[10] Cracknell, A.P., Introduction to Remote Sensing, Second Edition. CRC Press, 1991.

[11] Jaselskis, E.J., Gao, Z. \& Walters, R.C., Improving Transportation Projects Using Laser Scanning. Journal of Construction Engineering and Management, 131(3), pp. 377-384, 2005.

[12] Leica Geosystems, http://www.leica-geosystems.com

[13] Xiao, Y., Zhan, Q. \& Pang, Q., 3D Data Acquisition by Terrestrial Laser Scanning for Protection of Historical Buildings. 2007 International 
96 Building Information Modelling (BIM) in Design, Construction and Operations

Conference on Wireless Communications, Networking and Mobile Computing, IEEE, pp. 5966-5969, 2007.

[14] Tse, R.O.C., Gold, C. \& Kidner, D., 3D City Modelling from LIDAR Data. Advances in 3D Geoinformation Systems, Springer Berlin Heidelberg, Lecture Notes in Geoinformation and Cartography, chapter 10, pp. 161-175, 2008.

[15] Jiang, R., Jáuregui, D.V. \& White, K.R., Close-range photogrammetry applications in bridge measurement: Literature review. Measurement, 41(8), pp. 823-834, 2008.

[16] Al-kheder, S., Al-shawabkeh, Y. \& Haala, N., Developing a documentation system for desert palaces in Jordan using 3D laser scanning and digital photogrammetry. Journal of Archaeological Science, 36(2), pp. 537-546, 2009.

[17] Agnello, F. \& Brutto, M.L., Integrated surveying techniques in cultural heritage documentation.

[18] Yastikli, N., Documentation of cultural heritage using digital photogrammetry and laser scanning. Journal of Cultural Heritage, 8(4), pp. 423-427, 2007.

[19] Zender, H., Martínez Mozos, O., Jensfelt, P., Kruijff, G.J. \& Burgard, W., Conceptual spatial representations for indoor mobile robots. Robotics and Autonomous Systems, 56(6), pp. 493-502, 2008. 